\title{
Kontextabhängigkeit international vergleichbarer Arbeitslosenquoten
}

\author{
Hans Peter Litz \\ Institut für Volkswirtschaftslehre II und Statistik \\ Carl von Ossietzky Universität Oldenburg
}

Zusammenfassung: Die Daten zur registrierten Arbeitslosigkeit sind aufgrund national unterschiedlicher Konzepte für den internationalen Vergleich nicht brauchbar. An einer Reihe von Beispielen läßt sich belegen, daß auch die „standardisierte“ Arbeitslosenquote der OECD und die „vergleichbare“ Arbeitslosenquote von Eurostat nicht nur die nationalen Arbeitsmarktungleichgewichte widerspiegeln, sondern darüber hinaus auch die jeweiligen nationalen Rahmenbedingungen. Ob eine Person als arbeitslos erfaßt wird, ist oft weniger von ihrer tatsächlichen Situation als von ihrem formalen Status im jeweiligen Untersuchungskontext abhängig. Als dessen Dimensionen sind sowohl die Verfaßtheit des Arbeitsmarktes wie die Maßnahmen der nationalen Arbeitsmarktpolitik, die jeweiligen Systeme der sozialen Sicherheit und die Ausgestaltung der Aus- und Weiterbildungssektoren zu reflektieren. Im Folgenden wird diese Hypothese der Kontextabhängigkeit der Daten an ausgewählten Beispielen diskutiert. Zur Quantifizierung des Einflusses der verschiedenen kontextuellen Ebenen wird ein modulares System der latenten und der manifesten Arbeitslosigkeit entwickelt, dessen Bausteine mit den Berichtssystemen zur registrierten wie zur standardisierten Arbeitslosigkeit zu verbinden sind.

\begin{abstract}
Owing to distinctions in national concepts, the data on registered unemployment cannot be used for international comparisons. With a suitable set of examples, it can be demonstrated that even the standardized unemployment rate of the OECD and the comparable jobless rate of Eurostat based on ILO recommendations reflect not only the national imbalances in the labour market, but also the corresponding national labour environments. Whether particular persons are registered as unemployed frequently depends less on their actual personal situations than on their formal status within the relevant context under investigation. The applicable parameters include the conditions prevailing in the respective labour market, the measures of national labour policy, the diverse systems of social security, and the organization of the initial education and further training sectors. In this paper, the hypothesis of the context dependency of unemployment data is discussed with the aid of selected examples. To quantify the influence of the various contextual levels, a modular system comprising the latent and the manifest unemployment is developed, the building blocks of which have to be coupled with the reporting systems for both registered and standardized unemployment.
\end{abstract}

Schlüsselwörter: Standardisierte Arbeitslosenquote, ILO-Konzept der Statistik der Arbeitslosigkeit. 


\section{Die Statistik der Arbeits- und Erwerbslosigkeit in Europa}

\subsection{Statistische Konzepte zur Erfassung der registrierten Arbeitslo- sen und zur Erhebung der Erwerbslosen innerhalb der Labour- Force}

Die Bedeutung der monatlichen Arbeitslosen- bzw. Erwerblosenquoten als nationale Arbeitsmarkt- und Konjunkturindikatoren und als international vergleichbare Erfolgskriterien nationaler Wirtschafts- und Sozialpolitik steht außer Frage. Das Dilemma der derzeitigen europäischen Arbeitslosen-/Erwerbslosenstatistik besteht allerdings darin, daß die meisten nationalen Quoten und die ,,international vergleichbaren“ Quoten der OECD und von Eurostat auf begrifflich, methodisch und konzeptionell unterschiedlichen Ansätzen basieren und deshalb nicht aufeinander bezogen werden können.

Die von den nationalen Arbeitsverwaltungen (in Deutschland: Bundesanstalt für Arbeit; in Österreich: Arbeitsmarktservice) monatlich veröffentlichten Angaben zur Anzahl und Quote der Arbeitslosen betreffen die amtlich registrierten bzw. anspruchsberechtigten Arbeitslosen. Oft sind dies sogar die offiziellen Quoten (so z.B. in Deutschland und Österreich). Fast überall bestimmen deren monatliche Meldungen das öffentliche Bild von Arbeitslosigkeit sowie die nationale wirtschafts- und sozialpolitische Diskussion.

\subsubsection{Erfassung der registrierten / gemeldeten Arbeitslosen}

Die Registerauszählungen der nationalen Arbeitsverwaltungen stellen eine kostengünstige Totalerfassung der Betroffenen dar. Ihre Ergebnisse sind innerhalb weniger Wochen sowie in einem kleinräumlichen Maßstab verfügbar. Allerdings sind die Daten von den länderspezifischen Regulatorien bezüglich des betroffenen Personenkreises (Anspruchsberechtigung, Verfügbarkeit, Vermittelbarkeit) und von den jeweiligen nationalen Kriterien der Registerführung (Rückmeldung, Aktivitätsnachweis) geprägt. Diese nationalen amtlichen Daten weisen deshalb im europäischen Spektrum sehr große inhaltliche Unterschiede auf, so daß sie schon vor der Konzipierung der ,international vergleichbaren“ Arbeitslosenquoten durch die OECD und Eurostat in den 70er und 80er Jahren nur mit Vorbehalten und unter Berücksichtigung der jeweiligen Besonderheiten für den internationalen Vergleich herangezogen werden konnten. Mehrfach (1977, 1982 und 1987) wurden deshalb vom SAEG (Statistisches Amt der Europäischen Gemeinschaft) / Eurostat in Zusammenarbeit mit dem IAB (Institut für Arbeitsmarkt- und Berufsforschung, Nürnberg) Synopsen ihrer verschiedenen Definitionen und Erhebungskonzepte erstellt. ${ }^{1}$

\subsubsection{Erfassung der Erwerbslosen nach dem Erwerbskonzept (Labour-Force-Kon- zept)}

Die von der OECD und von Eurostat seit 1983 für die Länder der EU veröffentlichten, sogenannten „,standardisierten“ bzw. „,vergleichbaren“ Erwerbslosenquoten ${ }^{2}$ beziehen sich auf die von den nationalen Statistischen Ämtern im Rahmen der europäischen Arbeitskräfteerhebung (AKE) nach dem Labour-Force-Konzept der ILO (International Labour 
Office, vgl. Hussmanns et al., 1992) in Haushaltsstichproben ermittelten Erwerbspersonen. Diese umfassen alle Personen, die üblicherweise einer auf Erwerb ausgerichteten Tätigkeit nachgehen, unabhängig vom Umfang, d.h. mindestens eine Stunde pro Woche und vom Ertrag, also auch bei unbezahlter Tätigkeit von z.B. mithelfenden Familienangehörigen (vgl. Eurostat, 1998; OECD, 1997; Recktenwald, 1997). Die Resultate der Haushaltsbefragungen basieren auf der subjektiven Einschätzung der Befragten. Erwerbslosigkeit ist unter der Bedingung der Zugehörigkeit zur Labour-Force dann gegeben, wenn die Befragten in der Berichtswoche selbst nicht erwerbstätig waren, dem Arbeitsmarkt für eine neue Erwerbstätigkeit zur Verfügung standen und eigene Anstrengungen unternahmen, eine neue Erwerbstätigkeit zu finden. ${ }^{3}$ Erwerbslose sind folglich Personen, die sich aktiv um eine Arbeitsstelle bemühen, unabhängig davon, ob sie bei der Arbeitsplatzverwaltung als Arbeitslose gemeldet sind. Die einheitliche definitorische Abgrenzung des Sachverhalts der Erwerbslosigkeit in allen Ländern stellt dabei die Basis der internationalen Vergleichbarkeit der Ergebnisse dar. Für Deutschland und Österreich fallen diese Daten als Ergebnisse einer Unterstichprobe der Mikrozensen an. Aus den Mikrozensen selbst ermitteln die statistischen Ämter bei abweichenden Abgrenzungen der Erwerbslosen und der Erwerbspersonen eigene Erwerbslosenquoten ${ }^{4}$, die in der Vergangenheit bis zu zwei Prozent-Punkte über den Eurostat-Quoten lagen. Die Haushaltsbefragungen werden nur in Ausnahmefällen monatlich, in der Regel vierteljährlich (so z.B. in Österreich) oder jährlich (so in Deutschland) als 0,1 - 1 prozentige Stichproben durchgeführt. Zudem liegen sie wegen des zu großen Stichprobenfehlers nicht auf kleinräumlicher Ebene vor. ${ }^{5}$ Bis zur Veröffentlichung der erhobenen Daten vergehen oft mehrere Monate.

Die konzeptionelle Entwicklung, nationale Umsetzung und internationale Harmonisierung der zu ihrer Erfassung von Eurostat entwickelten Erhebungs- und Aufbereitungsprogramme unterlag in den letzten beiden Jahrzehnten einer kontinuierlichen methodischen Reflexion und Verfeinerung. Dabei lag das Schwergewicht in den 70er und 80er Jahren auf der internationalen Vereinheitlichung der definitorischen Grundlagen seitens der ILO (vgl. Hussmanns et al., 1992) und führte zu den international vergleichbaren Konzepten der ,standardisierten“ Erwerbslosenquoten von Eurostat und der ,vergleichbaren“ Erwerbslosenquote der OECD (vgl. Eurostat 1998). Die Einführung von monatlichen oder vierteljährlichen Arbeitskräftezählungen in einigen Ländern der EU Anfang und Mitte der 90er Jahre, so vor allem in Großbritanien (vgl. Working Party, 1995; Steel, 1997), gab den Anstoß zur Weiterentwicklung der Arbeitskräfteerhebung (AKE) von Eurostat in Richtung auf eine kontinuierliche Erhebung mit einem vierteljährlichen Berichtsturnus (vgl. Recktenwald, 1997, und van Bastelaer, 1998).

Ein aktuelles methodisches Problem stellen die von Eurostat oder der OECD ausgewiesenen monatlichen Statistiken dar. Sie ergeben sich für die zurückliegenden Zeiträume als Interpolationen von erhobenen Jahres- bzw. Vierteljahreswerten und für die Monate am aktuellen Rand als Extrapolationen der letzten verfügbaren Erhebungsdaten. Dabei unterscheiden sich die Verfahren von Eurostat und OECD sowohl hinsichtlich der Konzepte wie hinsichtlich ihrer Umsetzung. Die von Eurostat veröffentlichten Inter- und Extrapolationen basieren auf national unterschiedlichen „besten monatlichen Indikatoren der Erwerbslosigkeit", für die bzgl. Deutschlands auf die registrierten Arbeitslosen zurückgegriffen wird $^{6}$ (vgl. Eurostat 1998, S. 14). Die Berechnungen werden von Eurostat durchgeführt. Die monatlichen Daten der OECD werden von den nationalen statisti- 
schen Ämtern selbst erstellt, wobei sich die Konzepte sowohl innerhalb der Länder wie gegenüber den Eurostat-Konzepten für die jeweiligen Länder unterscheiden können. ${ }^{7}$ Eine vergleichende, detaillierte Darstellung der unterschiedlichen Interpolationsverfahren fehlt bisher sowohl für die Eurostat-Daten wie für die der OECD.

\subsection{Zur Komplementarität der Erwerbs- und Arbeitslosenstatistik}

Unter der Voraussetzung, daß die nationalen Registerdaten und die supranational standardisierten Stichprobenergebnisse definitorisch, konzeptionell und organisatorisch aufeinander bezogen werden können, könnte man die Existenz zweier Berichtssysteme als sinnvolle „Arbeitsteilung“ zwischen amtlicher Statistik und Arbeitsverwaltung verstehen, mit der amtlichen Arbeitslosenquote als nationalem, monatlich und vor allem regional verfügbarem Arbeitsmarkt- und Konjunkturindikator und der Erwerbslosenquoten der OECD und von Eurostat als internationalem Effizienzkriterium nationaler Beschäftigungsstrategien und Wirtschaftsverfassungen. Sind also, um mit G. Biffl (1994) zu fragen, „Eine nationale und eine internationale Arbeitslosenquote: der Stein der Weisen?“ Ergebnisse von arbeitsteiligen Produktionsprozessen sind voraussetzungsgemäß einheitliche Produkte oder zumindest kompatible Komponenten. Wie aus den gerade beschriebenen Erhebungskonzepten hervorgeht, erfassen die beiden Ansätze jedoch sehr unterschiedliche Sachverhalte, die zu jeweils spezifischen Coverage-Problemen führen.

In den Daten der Arbeitsverwaltungen fehlen die, aus welchen Gründen auch immer nicht-registrierten aber doch aktiv arbeitssuchenden Arbeitslosen. Die Daten der amtlichen Statistik enthalten nicht die von den Arbeitsämtern registrierten Arbeitslosen mit geringfügiger Beschäftigung. Das wechselseitige Coverage-Problem ${ }^{8}$ ist die Ursache dafür, daß sich beide Konzepte sachlich nicht ergänzen können, aber auch dafür, daß das eine Konzept vermutlich auch langfristig nicht das andere ersetzen kann. So dominiert die kleinräumlich, monatlich und auch relativ kurzfristig aus den Registern der Arbeitsverwaltung verfügbare Arbeitslosenstatistik in den meisten Ländern, in denen sie geführt wird, nach wie vor die nationale Diskussion und ist deshalb z.T. auch noch im internationalen Vergleich präsent. Ebenso wird die Regionalpolitik auf europäischer Ebene weiterhin auf sie angewiesen sein. Auf sie wird auch in Zukunft nicht verzichtet werden können (so auch Biffl, 1997, S. 41). Dazu kommt noch ein gemeinsames Coverage-Defizit: beide Ansätze erfassen nicht die in der Literatur mit dem Begriff der ,discouraged worker“ belegten, nicht permanent aktiv arbeitssuchenden Personen, die jedoch bei sich bietender Gelegenheit eine Arbeit aufnehmen. Umgekehrt ist vermutlich in beiden Statistiken ein schwer zu quantifizierender, nach allgemeiner Auffassung jedoch wesentlich geringerer Anteil von Scheinarbeitslosen enthalten.

Die hier vorgetragenen Coverage-Probleme werden in der Literatur vielfach diskutiert und quantifiziert, im wesentlichen jedoch als konstitutionelles Defizit der Arbeitsund Erwerbslosenstatistik behandelt, dem im Grunde nur durch ergänzende Berechnungen einer „Stillen Reserve“ (Institut für Arbeitsmarkt- und Berufsforschung, Brinkmann et al., 1987) oder einer „verdeckten Arbeitslosigkeit“ (Sachverständigenrat, 1998, S. 88 f) bzw. mit Hilfe zusätzlicher Konzepte einer „breiten Arbeitslosigkeit“ (OECD, 1996) oder einer „erweiterten Arbeitslosigkeit“ (Beschäftigungsobservatorium, Trends Nr. 30, 1998, S. 6 ff) möglicherweise sogar nur durch ein Bündel von „Indikatoren zur Arbeits- 
losigkeit“ (Bureau of Labour Statistics, Sorrentino, 1993) begegnet werden kann. Nur für diese zuletzt angeführten Indikatoren, die:

U 1: Quote der Langzeitarbeitslosen,

U 2: Übergangsquote in die Arbeitslosigkeit,

U 3: Quote der erwachsenen Arbeitslosen,

U 4: Quote der Vollzeitarbeitslosen,

U 5: Konventionelle Arbeitslosenquote,

U 6: Unterbeschäftigtenquote und

U 7: $\quad$ Arbeitslosenquote einschließlich der stillen Reserve

werden vom Bureau of Labor Statistics für die wichtigsten Industrienationen seit 1976 kontinuierlich empirische Daten berechnet.

Alle diese Ergänzungen bzw. Korrektive formulieren eine implizite Kritik nicht nur an der Statistik der registrierten Arbeitslosigkeit, sondern ebenso an der Erfassung der Erwerbslosigkeit nach dem ILO-Konzept ${ }^{9}$, ohne sich mit deren Defiziten explizit auseinander zu setzen oder gar auf der Basis ihrer systematischen Analyse konzeptionelle Verbesserungen vorzuschlagen. Einige kritische Anmerkungen in neueren Veröffentlichungen lassen darauf schließen, daß jedoch auch diese grundlegenden definitorischen und konzeptionellen Defizite in der methodologischen Diskussion wieder stärker Beachtung finden. Allerdings werden die Bedenken meist sehr allgemein und die Lösungsvorschläge eher perspektivisch formuliert. Ihr Spektrum reicht von der Notwendigkeit sinnvollerer Definitionen zur Messung der Arbeitslosigkeit ${ }^{10}$ bis zur Einforderung neuer theoretischer Bezugsrahmen ${ }^{11}$. Die Kritik beinhaltet auch Zweifel an der tatsächlichen Vergleichbarkeit der Quoten ${ }^{12,13}$, zumal sich auch konkrete Hinweise auf nationale Divergenzen bezüglich der instrumentellen Aspekte, insbesondere der Fragebogengestaltung und Frageformulierung (vgl. van Bastelaer, 1994), finden.

\section{Die internationale Vergleichbarkeit der Arbeitslosen- quoten der OECD und von Eurostat}

Die ,standardisierten“ bzw. ,,vergleichbaren“ Arbeitslosenquoten ${ }^{14}$ basieren auf dem Paradigma, daß die mittels eines einheitlichen Begriffs- und Methodenrasters erfaßte Daten per se, also ohne Berücksichtigung der jeweiligen Rahmenbedingungen vergleichbar seien. Eine Ausblendung der nationalen Kontexte ist jedoch nur statthaft, solange diese in den für die Statistik wesentlichen Aspekten weitgehend homogen sind (vgl. dazu und zum Folgenden auch Biffl, 1997, S. 49). Länderspezifische Unterschiede in der Auflösung des „Normalarbeitsverhältnisses“, unterschiedliche Strukturen im Bildungswesen und in den sozialen Sicherungssystemen wie die nationale Ausdifferenzierung aktiver und passiver Arbeitsmarktpolitiken lassen Zweifel daran aufkommen, daß die nationalen Erhebungen der Erwerbslosigkeit nach dem ILO-Konzept zu kontextunabhängigen Ergebnissen führen. ${ }^{15,16}$ 


\subsection{Die Problematik unterschiedlicher Erhebungs-Designs}

Auf die Notwendigkeit, sich die nationalen Erwerbslosendaten vor den spezifischen nationalen Hintergründen nochmals genauer anzusehen, verweist schon die bereits angesprochene Studie des niederländischen Statistikers van Bastelaer aus dem Jahre 1994 (van Bastelaer, 1994). In einer Analyse der Erfassung der Erwerbstätigkeit in den europäischen Arbeitskräfteerhebungen stellt er substantielle Unterschiede in der nationalen Fragebogengestaltung, insbesondere in der Anordnung und Ausformulierung der Fragen fest, die ihn zum Schluß kommen lassen, das gesamte Survey-Design der EU-LabourForce-Stichprobe müsse auf seine Response-Validität überprüft werden.

Diese sieht er vor allem bei der Erfassung der Aktivitäten am Rande der Erwerbsgesellschaft, wie etwa von geringfügiger Beschäftigung, Gelegenheitsarbeit und unbezahlte Tätigkeit von mithelfenden Familienangehörigen gefährdet. Bei deren Quantifizierung ist sowohl die Anordnung der Fragen, wie ihre Formulierung, u.U. sogar die explizite Nennung der ausgeübten Form einer marginalen Erwerbstätigkeit ausschlaggebend dafür, ob sich die Betroffenen den Erwerbspersonen zurechnen oder nicht. Da der Anteil dieser randständigen Kategorien von Erwerbspersonen in den Ländern der EU zwischen 1 \% und $10 \%$ schwankt, sind die fragebogenbedingten Unterschiede ihrer Erfassung nach van Bastelaer nicht trivial und für die internationale Vergleichbarkeit der Ergebnisse wesentlich. Wenn sich nun diese Erwerbstätigengruppe nur durch eine aufwendige Fragebogengestaltung identifizieren läßt, um wieviel zielgerechter müssen die Fragen formuliert werden, damit diese Personen als arbeitslose identifiziert werden können, wenn sie diese marginale Tätigkeit verlieren.

Die Schwierigkeiten der adäquaten Erfassung marginaler Beschäftigungsverhältnisse in ihren unterschiedlichen quantitativen nationalen Bedeutungen, wie in ihren divergierenderen nationalen Erscheinungsformen verdeutlichen die Notwendigkeit einer Vereinheitlichung des Erhebungsinstrumentariums bis ins Detail (vgl. dazu auch Recktenwald 1997, S. 79 f). Die Gesamtproblematik der laufenden Arbeitskräfteerhebung weist allerdings über eine ausschließlich formale Behandlung des Vereinheitlichungspostulats hinaus.

\subsection{Die Relevanz des Labour-Force-Konzepts für die Arbeitslosen- statistik angesichts der Auflösung von Normalarbeitsverhältnis- sen}

Van Bastelaer führt weiter aus (ebenda, S. 196), daß der Anteil geringfügiger Beschäftigungsverhältnisse von 10 und weniger Wochenstunden im europäischen Durchschnitt bei 1 - $2 \%$ liegt, aber bei 5 - $6 \%$ in Dänemark und im Vereinigten Königreich sowie bei $10 \%$ in den Niederlanden. Da sich in Ländern mit einem nennenswerten Arbeitsangebot im Bereich geringfügiger Beschäftigung das gesamte angebotene Arbeitsvolumen auf mehr Personen aufteilt, ist zu erwarten, und die Zahlen belegen dies auch, daß dort die Arbeitslosigkeit niedriger ist als in den anderen Ländern. Von einer echten Reduktion der Arbeitslosigkeit kann jedoch nur in dem Maße gesprochen werden, in dem diese geringfügige Beschäftigung freiwillig ist. ${ }^{17}$ Während in Ländern mit eher herkömmlichen Arbeitsmarktstrukturen der Verlust der Arbeit im allgemeinen eine existentielle Verände- 
rung der Lebens- und Arbeitssituation mit sich bringt, kann in Ländern mit deregulierteren Arbeitsbeziehungen eine existentielle Reduktion der Arbeitszeit um z.B. 30 Stunden, statistisch gesehen, nach wie vor mit dem Status „Erwerbstätigkeit“" verknüpft sein und ein Zugang zum Status Arbeitslosigkeit aus dem Verlust eines geringfügigen Beschäftigungsverhältnisses von im Extremfall einer einzigen Stunde resultieren. So verändern sich in diesen Fällen mit den strukturellen Bedingungen der jeweiligen nationalen Arbeitsmärkte die Bedeutungsgehalte der statistischen Informationen zur Erwerbstätigkeit bzw. -losigkeit.

Die Problematik eines rein formalen Umgangs mit den Erfassungskriterien zeigt J. Velling (1994) am Beispiel der „Kurzarbeit ,Null““ für die neuen Bundesländer Deutschlands auf. Diese Maßnahme nach dem Arbeitsförderungsgesetz hatte zur Folge, daß sich dort die Arbeitslosenquote nach dem ILO-Konzept 1991 nur auf 9,3\% belief, weil die Betroffenen aufgrund formal weiterbestehender Arbeitsverträge als Erwerbstätige gezählt wurden. Bei einer Erfassung dieses Personenkreises als Arbeitslose errechnet Velling eine Quote von 15,0 \% (ebenda, S. 13).

G. Schmid (1997, S. 19) belegt für die Niederlande eine weitere kontextuelle Besonderheit, die das Ausmaß der dort gemessenen Arbeitslosigkeit tangiert: Zwischen 1970 und 1994 hat sich die Anzahl der wegen Erwerbs- bzw. Berufsunfähigkeit vorzeitig verrenteten Personen im Verhältnis zur Zahl der Erwerbspersonen verdreifacht, wohingegen dieses Verhältnis in Deutschland in etwa dem gleichen Zeitraum konstant geblieben ist. In Deutschland liegt die Anzahl der von Erwerbs- oder Berufsunfähigkeit Betroffenen bei etwa $80 \%$ der Bezieher der übrigen sozial- und arbeitsmarktpolitisch begründeten Leistungen, während in den Niederlanden die erste Gruppe von Leistungsbeziehern die zweite um fast das Doppelte übertrifft. Dieser Sachverhalt läßt sich kaum anders als über eine spezifische niederländische Öffnung dieser Verrentungsform für (langfristig) Arbeitslose erklären. Diese in ihrem Ausmaß für Europa untypische Form der Entlastung des Arbeitsmarktes reduziert die niederländische Arbeitslosenquote weiter, so daß deren Höhe und Entwicklung im letzten Jahrzehnt mindestens ebenso stark von strukturellen Veränderungen des Arbeitsmarktes wie einer arbeitsmarktpolitisch ausgerichteten Sozialpolitik geprägt ist. ${ }^{18}$

\subsection{Die Auswirkungen des Labour-Force-Konzepts auf die Arbeitslo- senstatistik angesichts divergenter arbeitsmarktpolitischer Kon- texte}

In den Studien zum internationalen Vergleich von Indikatoren zur Arbeitslosigkeit diskutiert Sorrentino weitere kontextuelle Einflußfaktoren auf die Arbeitslosenstatistik (Sorrentino, 1993, S. 17 f und 1995, S. 43 ff). Am Beispiel Schwedens veranschaulicht sie, wie zumindest bis zu Beginn der 90er Jahre die Arbeitslosenquote durch eine aktive Arbeitsmarktpolitik beträchtlich nach unten gedrückt werden konnte. ${ }^{19}$ Erst als auch die Teilnehmer an Weiterbildungs- und Arbeitsbeschaffungsmaßnahmen nicht mehr dauerhaft vom Arbeitsmarkt absorbiert werden konnten, näherte sich die Quote dem EU Durchschnitt an. Inzwischen gehören derartige Maßnahmen, wenn auch in unterschiedlichem Umfang, zum gängigen sozialpolitischen Instrumentarium aller europäischen Staaten. In der Regel findet sich in ihnen Konglomerate von schulischer Aus- und Weiterbildung, 
„training on the job“ und lohnsubventionierter Beschäftigung. Unabhängig von der Gesamtwochenstundenzahl und der Zeitdauer der Maßnahme sind die Teilnehmer nach dem Labour-Force-Konzept als Erwerbstätige zu klassifizieren, wenn der faktische Anteil an praktischer Arbeit auch nur eine Stunde beträgt. Auch hier stellt sich die Frage, ob damit der Bedeutungsgehalt des Begriffs „Erwerbstätigkeit“ richtig getroffen ist.

Steht hier, wie am Beispiel geringfügiger Beschäftigung, die Trennschärfe des EinStunden-Kriteriums zur Diskussion, so lenkt ein weiteres Beispiel von Sorrentino den Blick auf die Angemessenheit des Kriteriums aktiver Arbeitsplatzsuche. In Italien, führt sie aus, dauern die Personalauswahlverfahren sehr lange. So warten die Bewerber für Tätigkeiten im öffentlichen Dienst z.T. länger als ein Jahr auf einen Bescheid. Wenn sie mangels Alternativen keine weiteren Versuche unternehmen, einen anderen Arbeitsplatz zu suchen, werden sie nach dem Labour-Force-Konzept als Nichterwerbspersonen erfaßt (Sorrentino, 1995, S. 43). Ähnliches widerfährt seit einigen Jahren in Deutschland den beim Arbeitsamt als arbeitslos Gemeldeten. Wenn sie mangels realistischer alternativer Bewerbungsformen sich auf das Arbeitsamt verlassen und keine weiteren Aktivitäten nachweisen können, werden sie in der AKE nicht mehr als Arbeitslose erfaßt (vgl. dazu auch Velling, 1994, S. 18).

Die Kontextrelevanz sogar kultureller Rahmenbedingungen läßt sich anhand japanischer Erfahrungen belegen. Dort signalisiert eine außergewöhnlich geringe Arbeitslosenquote der weiblichen Arbeitnehmerinnen, daß sich diese bei Verlust eines Arbeitsplatzes eher vom Arbeitsmarkt zurückziehen als sich arbeitslos zu melden oder aktiv nach Arbeit zu suchen (Sorrentino, 1995, S. 43). Nicht zuletzt stehen die nationalen Arbeitsmärkte über die internationale Arbeitsmigration in einem Zusammenhang, so daß auch allgemeine ausländerpolitische Prinzipien der Länder, etwa im Falle von Arbeitslosigkeit, die nationalen Arbeitslosenquoten tangieren können (Sorrentino, 1993, S. 18).

\section{3 Überlegungen zu einem Indikatorensystem zur Erfas- sung von Arbeitsmarktdefiziten}

\subsection{Empirische und theoretische Aspekte der Kontextabhängigkeit international vergleichbarer Arbeitslosenquoten}

Die angeführten konzeptionellen Defizite der „,vergleichbaren“ Arbeitslosenquote sind bisher weder so umfassend recherchiert, daß alle relevanten kontextuellen Dimensionen benennbar wären, noch so systematisch aufgearbeitet, daß die innere Struktur dieser Dimensionen erkennbar werden könnte. Insofern ist hier besser von der Hypothese oder der Vermutung einer Kontextabhängigkeit zu sprechen, die durch eine breitere empirische Analyse zu widerlegen oder zu erhärten wäre. Allerdings läßt sich an den Fallbeispielen verdeutlichen, was mit der Kontextabhängigkeit der ,vergleichbaren“ Arbeitslosenquoten gemeint ist. Natürlich soll damit nicht das triviale Faktum angesprochen werden, daß das sozio-ökonomische Umfeld die Strukturen und die Entwicklung des zu erfassenden Sachverhalts beeinflußt, also daß z.B. eine Verlängerung der allgemeinen Schulpflicht sowohl die Jugenderwerbstätigkeit wie die Jugendarbeitslosigkeit tangiert.

Unter Kontextabhängigkeit ist hier einerseits die Reagibilität der Meßergebnisse auf 
eine Veränderung der Rahmenbedingungen zu verstehen, die den realen Status der Erhebungsobjekte unverändert läßt und, wie im Fall vorzeitiger oder sachlich ungerechtfertigter Verrentung, nur den formalen Status betrifft. Umgekehrt, wie im Falle der Kurzarbeit „Null“, ist auch die Stabilität der Daten bei einer substantiellen Veränderung der Situation der Betroffenen Ausdruck dieser Kontextabhängigkeit. Strikt formalistisch betrachtet, mißt die AKE die diskutierte Unterschiedlichkeit der Fragebögen einmal außer Acht gelassen den definierten Sachverhalt natürlich auf vergleichbare Weise. Die Frage ist nur, ob die Definition in allen Ländern gleichermaßen gültige Ergebnisse liefert.

Im Kern läuft die Behebung der Kontextabhängigkeit eines Erhebungskonzeptes auf ein Adäquationsproblem ${ }^{20}$ hinaus. Dieses besteht - kurz gefaßt - darin, zu prüfen, ob eine Meßvorschrift in einer konkreten Untersuchungssituation den zu messenden theoretischen, im Sinne M. Webers und der Adäquationstheorie als idealtypisch zu verstehenden Begriff auf adäquate Weise erfaßt. Internationale Vergleichbarkeit der Ergebnisse erfordert eine gleiche Trennschärfe der Meßkriterien in den unterschiedlichen nationalen Kontexten.

Die aufgezeigten Defizite der Arbeitslosenkonzepte von Eurostat und OECD lassen sich deshalb auch nicht durch die bereits angesprochenen Ergänzungen der Arbeitslosenstatistik in Form von Schätzungen einer ,,verdeckten“ (SVR), ,breiten“ (OECD) oder ,erweiterten“ (Beschäftigungs-Observatorium) Arbeitslosigkeit auffangen. Zum einen gilt, daß diese Alternativ-Rechnungen begrifflich und konzeptionell wenig fundiert sind und nur in Expertenkreisen wahrgenommen werden, also weder die öffentliche Diskussion noch den internationalen Vergleich wesentlich beeinflussen. Gravierender ist, daß in den Alternativ-Rechnungen auch Personen erfaßt werden, die entweder von der Höhe der Bezahlung oder der Arbeitsdauer als Voll- bzw. Teilzeiterwerbstätige bzw. von der Länge und Intensität der Weiterbildungsmaßnahme als Angehörige des Bildungssektors zu begreifen sind. ${ }^{21}$ Insofern gelten für diese Alternativen ähnliche Divergenzen zwischen formalem (statistischem) und realem Status wie bei den gebräuchlichen Konzepten zur Arbeitslosigkeit. Auch hier müßte von Kategorie zu Kategorie der Alternativ-Rechnung und in Abhängigkeit von den nationalen Rahmenbedingungen eine schärfere begriffliche Abgrenzung zwischen Arbeitslosen und Nichtarbeitslosen erfolgen.

Die Beispiele erlauben die Vermutung, daß das Labour-Force-Konzept, insbesondere das Ein-Stunden-Kriterium und das Kriterium der aktiven Arbeitsplatzsuche, den Veränderungen der Arbeitsmärkte und der Arbeitsmarktpolitiken nicht mehr angemessen ist. Das klassische Labour-Force-Konzept ist von einem produktionsorientierten Potentialbegriff bestimmt, nach dem alle, auch die geringfügigsten (potentiellen) Beiträge zur Produktion berücksichtigt werden sollen. Dies ist die zentrale Begründung für das Ein-StundenKriterium zur Erfassung der Erwerbstätigkeit (vgl. Hussmanns et al., 1992, S. 71). Die Konsequenzen dieser Meßvorschrift für die Erfassung der Arbeitslosigkeit werden von Hussmanns et al. nur kurz diskutiert und als wenig problematisch abgetan. ${ }^{22}$ Diese Einschätzung ist allerdings nur einer bestimmten Verfaßtheit des Arbeitsmarktes angemessen, die im wesentlichen durch Normarbeitsverhältnisse und höchstens Teilarbeitsverhältnisse (in der Regel für Frauen) geprägt ${ }^{23}$ und im allgemeinen nur friktionellen, saisonalen oder konjunkturellen Verwerfungen ausgesetzt ist. Andererseits sind die oft maßnahmeorientierten Konzepte der oben angesprochenen Alternativ-Rechnungen zu sehr an den institutionellen Aspekten der Organisation und Finanzierung der Programme orientiert 
und weniger am tatsächlichen Status des betroffenen Klientels auf dem Arbeitsmarkt. Mit diesem Argument soll, wie im nächsten Abschnitt dargelegt wird, auf eine statistische Erfassung der institutionellen Aspekte keinesfalls verzichtet werden.

Die Ablösung industriell geprägter (fordistischer) Produktionsprozesse, die Zunahme deregulierter Arbeitsverhältnisse und ein strukturelles Überangebot an Arbeit verlaufen weder zeitlich linear noch regional einheitlich. Sie konstituieren national unterschiedlich strukturierte Arbeitsmärkte, induzieren spezifische nationale Arbeitsmarktpolitiken und verändern auf unterschiedliche Weise die allgemeinen sozialen, politischen und kulturellen Rahmenbedingungen. Vor diesem Hintergrund verlieren auch die standardisierten Arbeitslosenquoten der OECD und von Eurostat und die Alternativ-Rechnungen ihre eindeutige internationale wirtschaftspolitische Signalfunktion und nationale sozialpolitische Relevanz. Aus praktischen und wirtschaftspolitischen Gründen ist es dennoch nicht empfehlenswert, das bisherige Meßkonzept durch ein einziges kontextneutrales zu ersetzen, sondern die unterschiedlichen kontextuellen Aspekte durch ein modulares System mit abzubilden.

\subsection{Die Entwicklung eines modularen Systems nationaler und inter- national vergleichbarer Arbeitslosenquoten}

Auf die Arbeitsmarktindikatoren, insbesondere auf die Statistik der Arbeitslosigkeit sind vielfältige und divergente Informationsbedürfnisse gerichtet. Nur grob umrissen, erstrekken sie sich auf die ökonomischen, beruflichen, sozialen und individuellen Dimensionen der Arbeitslosigkeit bei den Betroffenen, auf die finanziellen, organisatorischen und evaluativen Aspekte der eingesetzten arbeitsmarktpolitischen Maßnahmen und natürlich auf die rechtlichen, finanziellen, organisatorischen und quantitativen Koordinaten der mit Arbeitslosigkeit befaßten Institutionen, insbesondere der Arbeitslosenversicherungen. Auf allen drei Ebenen ist eine entsprechende sachliche Differenzierung der Daten sowie eine hinreichende Regionalisierung und Periodisierung notwendig. Vor diesem Hintergrund scheint es nicht sinnvoll und kaum realisierbar, eine (einzige) operationale Definition von Arbeitslosigkeit zu entwickeln. Erfolgsversprechender im Sinne der obigen Informationsaufgaben scheint es, die verschiedenen Aspekte über spezifische, statistische Komponenten darzustellen. ${ }^{24}$ Die Aufgaben des zu entwickelnden modularen System bestehen in der

- Verzahnung der Statistik der registrierten Arbeitslosigkeit mit der Statistik der Erwerbslosigkeit nach dem Labour-Force-Konzept,

- Schaffung einer gemeinsamen Plattform zur monatlichen und kleinräumlichen Berichterstattung auf der Basis von Registerauszählungen,

- Trennung des Nachweises einer (möglichst) präzise definierten, faktischen Arbeitslosigkeit von einer durch arbeitsmarktpolitische Maßnahmen aufgefangenen (früheren oder potentiellen) Arbeitslosigkeit und

- Abschätzung der verschiedenen, durch arbeitsmarktpolitische Maßnahmen tangierten Personengruppen, insbesondere der 
- in Arbeitsbeschaffungsmaßnahmen Befindlichen,

- in Weiterbildung und Umschulung Begriffenen,

- sich resignativ Zurückgezogenen (passive stille Reserve) und der

- vorzeitig in den Ruhestand oder die Erwerbsunfähigkeit Getretenen.

Die im Quantifizierungsprozeß zu präzisierenden Module beziehen sich auf die verschiedenen arbeitsmarktrelevanten Aktionsebenen (vgl. Abb. 1):

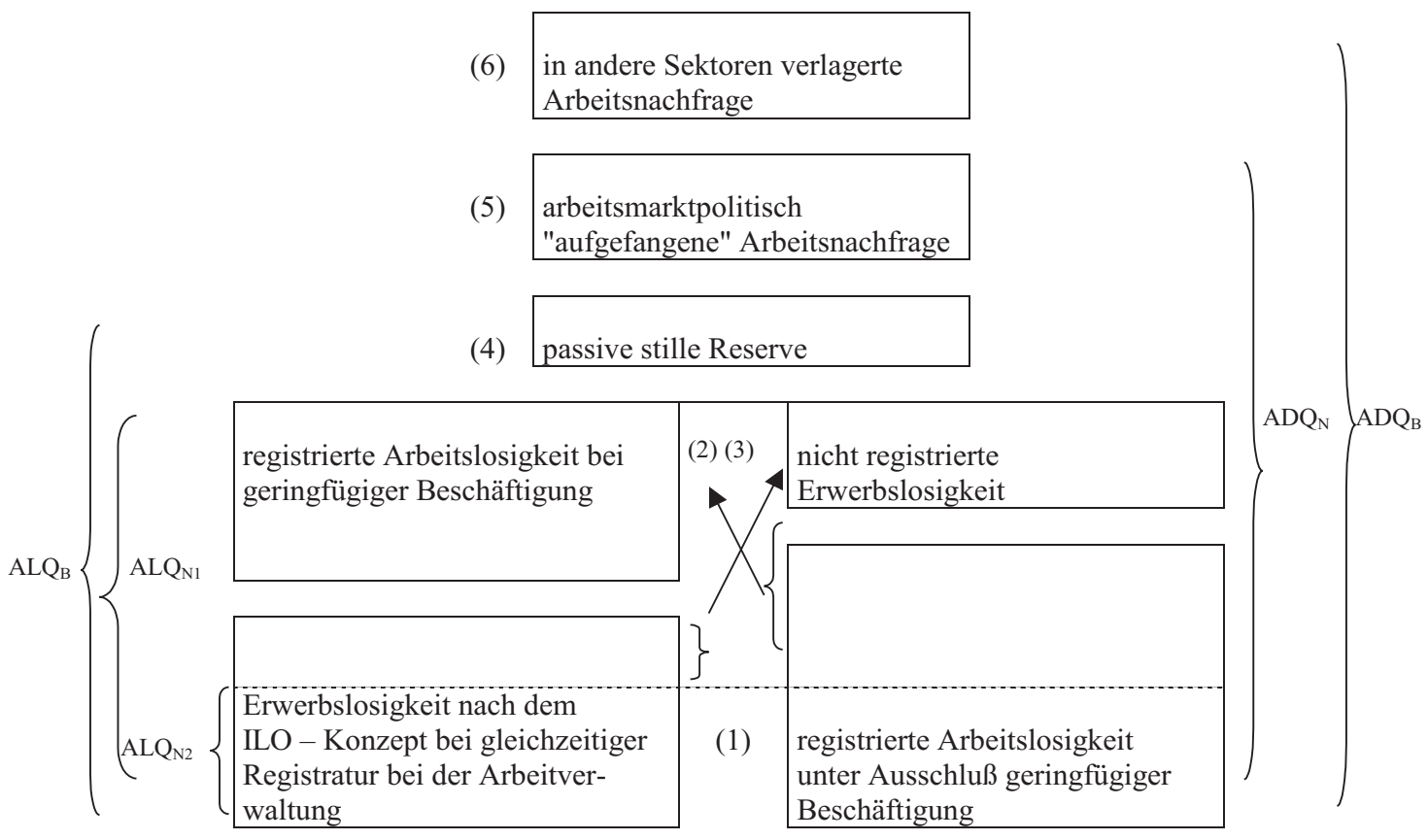

Anmerkungen zum sachlichen Gehalt und zur Erhebungsform der Module

(1) und (2): aus den Unterlagen der Arbeitsmarktverwaltung und der AKE (Arbeitskräfteerhebung von Eurostat): registrierte Arbeitslosigkeit

(3): $\quad$ aus der AKE (Regionalisierung problematisch): aktive stille Reserve ${ }^{26}$

(4): $\quad$ aus der AKE (Regionalisierung problematisch): passive stille Reserve

(5): aus den Unterlagen der Arbeitsverwaltung:

Personen in Maßnahmen der beruflichen Wiedereingliederung und der Weiterbildung mit einem Arbeits- oder Ausbildungsumfang von mehr als einem Drittel ${ }^{27}$ der regulären Arbeitszeit. Bei Unterschreiten der Grenze erfolgt eine Zuordnung zum Modul (2).

(6): $\quad$ aus den Unterlagen der Soz.Versicher.Träger und aus der AKE

Abbildung 1: Modulares System zur Statistik der Arbeitsmarktdefizite

Daraus resultieren:

- Eine unmittelbar dem Arbeitsmarkt zugeordnete Bruttoquote der Arbeitsmarktdefizite $\mathrm{ADQ}_{B}$ soll das Ausmaß an potentieller Arbeitslosigkeit widerspiegeln, das 
ohne aktive oder passive arbeitsmarktpolitische Maßnahmen aus der Arbeitsmarktentwicklung resultiert.

- Aus dieser Quote ${ }^{25}$ kann durch Subtraktion der Personen, die den Arbeitsmarkt aufgrund von Arbeitslosigkeit auf Dauer (z.B. infolge von Frühverrentung) oder zumindest mittelfristig (z.B. durch Rückkehr in den Bildungssektor, Abwanderung aus dem Erfassungsgebiet oder durch Übernahme einer mehrmonatigen bindenden Aufgabe im häuslichen Bereich) verlassen haben (Modul 6), eine Nettoquote der Arbeitsmarktdefizite $\mathrm{ADQ}_{N}$ der ohne passive Arbeitsmarktpolitik potentiell Arbeitslosen abgeleitet werden.

- Unter Herausrechnung eines Moduls 5 von temporär durch arbeitsmarktpolitische Aktivitäten aufgefangener Arbeitslosigkeit ergäbe sich schließlich die Bruttoarbeitslosenquote $\mathrm{ALQ}_{B}$ der aktiven und passiven Arbeitslosen.

- Die Nettoarbeitslosenquote $\mathrm{ALQ}_{N 1}$ entspräche der mengenmäßigen Vereinigung, die Nettoarbeitslosenquote $\mathrm{ALQ}_{N 2}$ dem mengenmäßigen Durchschnitt der bisherigen national registrierten und der international ,vergleichbaren“ Arbeitslosenquote.

Mit Ausnahme des Moduls (3), d.h. bei Vernachlässigung der Differenzierung zwischen den Quoten $\mathrm{ALQ}_{N 1}$ und $\mathrm{ALQ}_{N 2}$, könnten die Module im wesentlichen auf Registerdaten gestützt werden, d.h. die Daten könnten im Prinzip sowohl in monatlichen Abständen wie kleinräumlich zur Verfügung gestellt werden. Nur die Komponenten (3) und (4) müßten aus Jahresdaten monatlich und regional inter- bzw. extrapoliert werden.

Amtliche wirtschafts- und sozialstatistische Daten sollten nicht nur ihrem Gegenstand gegenüber adäquat sein, sondern möglichst auch den wirtschafts- und sozialpolitischen Aufgabenstellungen. Zur Beurteilung des Arbeitsmarktes und der Arbeitsmarktpolitik wie des arbeitsmarktrelevanten gesellschaftlichen Umfeldes sind alle Quoten heranzuziehen: Die Bruttoquote der Arbeitsmarktdefizite indiziert die generelle Funktionalität des Arbeitsmarktes und die langfristige Wirksamkeit der Arbeitsmarktpolitik. Aus dem Verhältnis der Bruttoquoten zur Nettoquote der Arbeitsmarktdefizite kann auf das gesellschaftliche Absorptionspotential im Falle von Arbeitslosigkeit geschlossen werden. Die Bruttoarbeitslosenquote gibt im Vergleich mit der Nettoquote der Arbeitsmarktdefizite Auskunft über den unmittelbaren Wirkungsgrad aktiver Arbeitsmarktpolitik. Unter sozialund arbeitsmarktpolitischen Aspekten stellt die Nettoarbeitslosenquote $\mathrm{ALQ}_{N 1}$ der aktiven Arbeitslosen die zentrale Orientierungsgröße dar. Für den von ihr erfaßten Personenkreis gibt es eine unmittelbare Nähe zum Arbeitsmarkt und einen artikulierten Bedarf an (zusätzlicher) Arbeit in erheblichem Umfang. ALQ $_{N 2}$ quantifiziert darüber hinaus die relative Bedeutung des ,harten Kern“, dessen Situation sowohl den Erfassungskriterien des ILO-Konzepts wie den Melde-, Aktivitäts- und Verfügbarkeitskriterien der Arbeitsverwaltung genügt. Das Verhältnis der beiden Nettoquoten könnte damit das individuelle Absorptionspotential im Falle von Arbeitslosigkeit ausdrücken.

Die nach wie vor bedeutende Rolle der Statistik der registrierten Arbeitslosen sowohl im nationalen Rahmen wie auch im internationalen Vergleich, sei es direkt oder indirekt als bester monatlicher Indikator, und nicht zuletzt die tragende Rolle der Register im modularen Konzept macht es erforderlich, sich auch wieder intensiver mit ihren nationalen Divergenzen auseinander zu setzen. Dabei sind die jeweiligen nationalen Richtlinien zur 
Abgrenzung des betroffenen Personenkreises und zur Führung der Register zu eruieren. Auch hier ist es notwendig, ihre Vergleichbarkeit vor dem Hintergrund der jeweiligen nationalen Verfaßtheit der Arbeitsmärkte, des Bildungswesens und der Systeme der sozialen Sicherheit sowie den Ausgestaltungen der jeweiligen nationalen Arbeitsmarktpolitik zu analysieren. Eine exakte Beschreibung der Erfassungsmodalitäten der registrierten bzw. anspruchsberechtigten Arbeitslosen dient auch der modularen Verzahnung der umseitig beschriebenen Komponenten der Brutto- und der Nettoquoten mit den amtlichen Arbeitslosenquoten. Sollte diese gelingen, wäre es möglich, die international vergleichbaren Quoten mit den monatlich und kleinräumlich von den Arbeitsverwaltungen ausgewiesenen Quoten in einem kohärenten System zu vereinen.

\section{Anmerkungen}

${ }^{1}$ Vgl. Koller und König (1977), Werner (1982) und Hitz (1987). Eine aktuelle Übersicht findet sich bei White und Leyland (1992); vgl. dazu auch Litz (1999).

${ }^{2}$ Üblicherweise werden auch diese Quoten als Arbeitslosenquoten bezeichnet. Die Verwendung des Begriffs „Erwerbslosenquote“ in diesem Abschnitt dient nur der besseren Unterscheidung der beiden konzeptionell verschiedenen Ansätze.

${ }^{3}$ Die Charakteristika der sofortigen bzw. kurzfristigen Verfügbarkeit und einer hinreichenden Aktivität bei der Arbeitssuche unterliegen ebenfalls der subjektiven Einschätzung der Befragten.

${ }^{4}$ Vgl. Bartunek (1994 und 1996), Statistisches Bundesamt (1998) und Mayer (1998).

${ }^{5}$ Kleinräumliche Daten unterliegen einem Stichprobenfehler, der um so größer ist, je kleiner der regionale Bevölkerungsumfang ist. Die Stichprobengröße der neu konzipierten, kontinuierlichen AKE sieht auf der regionalen Ebene von Nuts II (dies entspricht etwa der Größe eines mittleren Bundeslandes) für die Jahresdurchschnitte von $5 \%$ Bevölkerungsanteilen noch einen höchst zulässigen relativen Standardfehler von 8 \% vor (vgl. van Bastelaer, 1998, op.cit., S. 142).

${ }^{6}$ Für Österreich ist laut Eurostat (1998, S. 14) kein monatlicher Indikator verfügbar.

${ }^{7}$ Zukünftig ist von der OECD die Übernahme der Eurostat-Daten vorgesehen, vgl. Statistisches Bundesamt (1997), S. 8.

8 Aktuelle Daten zur teilweisen Quantifizierung der Überlappungen finden sich in Europäische Kommission (1999), Anhang: Gemeldete Arbeitslosenzahlen und harmonisierte Arbeitslosenzahlen, S. 143-148.

${ }^{9}$ Auch die ILO betont die Notwendigkeit, neben der Arbeitslosigkeit auch die Unterbeschäftigung zu erfassen, und schlägt dazu theoretische Konzepte zur Ermittlung der sichtbaren und unsichtbaren Unterbeschäftigung vor (vgl. Hussmanns et al., 1992, S. 121 145). Allerdings ist, abgesehen von Sorrentino, bisher keines der Konzepte auch nur ansatzweise aufgegriffen worden.

${ }^{10}$ M. Godfrey (1986) in einer Betrachtung der Arbeitsmarkttheorien unter dem Aspekt der Globalisierung.

${ }^{11}$ So Schmid (1997, S. 16), angesichts einer von der OECD ausgewiesenen Bandbreite der Arbeitslosenquote für die Niederlande zwischen 7 und $27 \%$.

${ }^{12}$ So wird z.B. im Gemeinschaftsgutachten der wirtschaftswissenschaftlichen Forschungs- 
institute von 1997 am Beispiel der Beschäftigten und der Arbeitslosen festgestellt, daß „die offiziellen nationalen wie auch supranationalen Arbeitsmarkt- und Beschäftigtenstatistiken ... mit einiger Vorsicht zu interpretieren (sind). Ihre Aussagefähigkeit ist schon deshalb eingeschränkt, weil ... Veränderungen am Arbeitsmarkt in den Definitionen nur unzureichend berücksichtigt werden“, DIW (1997), S. 839.

${ }^{13}$ In den Schlußfolgerungen ihres Beitrags „Erfassung der ,wahren“ Arbeitslosigkeit in Österreich“ formuliert und begründet G. Biffl ebenfalls Zweifel an der Vergleichbarkeit der international vergleichbaren Quoten, Biffl (1997, S. 49).

${ }^{14}$ Mit Blick auf den internationalen Vergleich und den allgemeinen Sprachgebrauch werden im Folgenden auch die Erwerbslosenquoten nach dem ILO-Konzept als Arbeitslosenquoten bezeichnet.

${ }^{15}$ Man muß sich nur eine alle Arbeitslose umfassende beschäftigungspolitische Maßnahme von hoher Attraktivität oder großem Nachdruck vorstellen. Diese würde selbst bei einem geringfügigen Beschäftigungsanteil von nur einer Stunde pro Woche die Erwerbslosenquote gegen Null bringen.

${ }^{16}$ Der hier grob umrissene Sachverhalt der Kontextbedingungen der Arbeitslosenstatistik wird von G. Schmid unter den Begriff der Beschäftigungsregimes gefaßt. Unter Beschäftigungsregimes mit den Komponenten Produktionsregime und Arbeitsmarktregime versteht er ,des Ensemble von Institutionen und der darauf einwirkenden Politik, die das Niveau von Arbeitslosigkeit und Beschäftigung simultan determinieren“ (vgl. G, Schmid, 1997, S. 16). Nur vor dem Hintergrund des theoretischen Rahmens dieser Regimes läßt sich seiner Auffassung nach das statistische Erscheinungsbild der Arbeitslosigkeit erklären.

17 Eine Analyse der, in einem Umfang von bis zu 15 Stunden Beschäftigten nach der gewünschten Arbeitszeit ergibt, daß nur bei knapp 50 \% der Befragten die tatsächliche der gewünschten Arbeitszeit entspricht. Etwa knapp ein Viertel der geringfügig Beschäftigten präferiert dagegen eine Arbeitszeit von mehr als 35 Stunden (Daten des Sozioökonomischen Panels, Welle N, 1998, eigene Auswertung).

${ }^{18}$ Einen negativen Einfluß der Frühverrentung auf die Aussagefähigkeit der niederländischen Arbeitslosenquoten konstatiert auch Schettkat (1997), S. 807.

191989 z.B. standen den 66 Tsd. erfaßten Arbeitslosen 145 Tsd. Personen in Arbeitsmarktprogrammen gegenüber, vgl. Sorrentino (1993, S. 17).

${ }^{20}$ Zum methodologischen Prinzip der Adäquation in der Wirtschaft- und Sozialstatistik vgl. Hartwig (1956), Grohmann (1985) und Litz (1990).

${ }^{21}$ Zum Konzept der erweiterten bzw. breiten Arbeitslosigkeit vgl. Beschäftigungsobservatorium, Trends Nr. 30, S. 6 f; zum Konzept der verdeckten Arbeitslosigkeit vgl. Sachverständigenrat, op.cit., S. 88 f, insbes. Tab. 35 .

22 ,Where data are available, it is usually found that the proportion of persons working, say, less than five hours a week does not exceed a few percentage points of the total employment. This means that raising the one hour criterion in the definition of employment by a few hour is in practice not likely to change substantially the resulting number of employed persons even over a period of time. However, the effects on unemployment statistics may be relatively more significant" (Hussmanns, 1992, S. 72).

${ }^{23} \mathrm{Da}$ die gesamte Statistik zu Erwerbstätigkeit noch weitgehend auf Normalarbeitsverhältnisse ausgerichtet ist und daß deshalb die Daten in überholte Schemata gepreßt werden, bemängelt auch die Kommission für Zukunftsfragen der Freistaaten Bayern und 
Sachsen, vgl. Kommission für Zukunftsfragen, 1997, S. 182.

${ }^{24}$ Eine „Ausdifferenzierung der Aggregate“ und ein statistisches „Baukastenkonzept für Arbeitslosigkeit und Erwerbstätigkeit" schlagen auch Biffl (1997, S. 49) bzw. Bartunek (1996, S. 363) vor.

${ }^{25}$ Die Nenner aller im folgenden definierten Quoten ergeben sich jeweils aus der Zahl der Erwerbspersonen nach dem ILO-Konzept zuzüglich der Module im Zähler der Quote (falls diese nicht schon bei den Erwerbspersonen mitgerechnet werden).

${ }^{26}$ Unter Einschluß unfreiwilliger geringfügiger Beschäftigung.

${ }^{27}$ Die genaue Grenze kann erst aufgrund der Analyse der in den Bezugsländern durchgeführten Maßnahmen festgelegt werden.

\section{Literatur}

Autorengemeinschaft. Der Arbeitsmarkt in der Bundesrepublik Deutschland in den Jahren 1997 und 1998. MittAB, Heft 1, 1998.

E. Bartunek. Berechnung von Arbeitslosenquoten in Österreich. Österr. Zeitschrift für Statistik und Informatik, 23 Jg., Heft 1, S. 13-20, 1994.

E. Bartunek. International vergleichbare Daten zur Arbeitslosigkeit in Österreich. Statistische Nachrichten, Nr. 5, S. 357-367, 1996.

A. van Bastelaer. Differences in Measurement of Employment in the Labour Force Surveys in the European Community. Journal of Official Statistics, Vol. 10, No. 3, S. $277-$ 305, 1994.

A. van Bastelaer. Eine kontinuierliche Arbeitskräfteerhebung für die Europäische Union. Austrian Journal of Statistics, Vol. 27, No. 3, S. 139-158, 1998.

Beschäftigungsobservatorium (Employment Observatory). MISEP Basisinformationsberichte. I.A.S., Institute for Applied Socio-Economics, Deutschland 1997 / UK 1997 / Dänemark 1997 / Niederlande 1997 / Österreich 1998, Berlin 1997 u. 1998.

Beschäftigungsobservatorium (Employment Observatory). Trends, Nr. 30. I.A.S., Institute for Applied Socio-Economics, Berlin 1998.

G. Biffl. Eine nationale und eine internationale Arbeitslosenquote: der Stein der Weisen. Österr. Zeitschrift f. Statistik und Informatik, 23 Jg., Heft 1, S. 3-7, 1994.

G. Biffl. Erfassung der „wahren“ Arbeitslosigkeit in Österreich. WIFO-Monatsberichte, Nr. 1, S. 41-50, 1997.

C. Brinkmann et al. Methodische und inhaltliche Aspekte der Stillen Rerserve. MittAB, S. 387-403, 1987.

DIW (Hrsg.). Zur Lage der Weltwirtschaft und der deutschen Wirtschaft im Herbst 1997. Wochenbericht, 44, 1997. 
Europäische Kommission. Beschäftigung in Europa 1998. Brüssel, 1999.

Eurostat. Erhebung über Arbeitskräfte, Ergebnisse 1997. Luxembourg, 1999.

Eurostat. Arbeitslosigkeit, Monatsbericht, Themenkreis 3. Reihe B, Nr. 9, 1998. Luxembourg, 1998.

M. Godfrey. Global Unemployment: The New Challenge to Economic Theory. Harvester Press, Brighton, 1986.

H. Grohmann. Vom theoretischen Konstrukt zum statistischen Begriff. Das Adäquationsproblem. Allg. Stat. Archiv, Bd. 69, S. 1-15, 1/1985.

H. Hartwig. Naturwissenschaftliche und sozialwissenschaftliche Statistik. Zeitschr. f. $d$. ges. Staatsw., Heft 12, S. 252-266, 1956.

H. Hitz. Erwerbsstatistische Praxis und die Ermittlung von Arbeitslosenquoten im internationalen Vergleich. BeitrAB, Nr. 97, Nürnberg, 1987.

R. Hussmanns, F. Mehran, and V. Verma. Surveys of economically active population, employment, unemployment and underemployment, an ILO manual on concepts and methods. 2. Aufl., International Labour Office, Geneva, 1992.

M. Koller und J. König. Internationaler Vergleich der Arbeitslosenquoten. BeitrAB, Nr. 29, Nürnberg, 1977.

Kommission für Zukunftsfragen der Freistaaten Bayern und Sachsen. Erwerbstätigkeit und Arbeitslosigkeit in Deutschland Entwicklung, Ursachen und Maßnahmen, Teil III, Maßnahmen zur Verbesserung der Beschäftigungslage. Bonn, 1997.

H.P. Litz. Statistische Adäquation und Idealtypus, Anmerkungen zur Methodologie der Wirtschafts- und Sozialstatistik. Allg. Stat. Archiv, Bd. 74, 4, S. 429-456, 1990.

H.P. Litz. Nationale und supranationale Konzepte der Messung der Arbeitslosigkeit und Probleme des internationalen Vergleichs. In W.v. Bülow et al., Hrsg., Globalisierung und Wirtschaftspolitik, S. 159-182. Metropolis Verlag, Marburg, 1999.

L. Mayer. Erwerbslosigkeit 1987 - auch im internationalen Vergleich, Ergebnisse des Mikrozensus und der EG-Arbeitskräftestichprobe. WiSta, 12, S. 849-863, 1988.

OECD. Economic Survey 1995, Netherlands. Paris, 1996.

OECD. Statistics Directorate. Main Economic Indicators, Sources and Definitions. Paris, 1997.

J. Recktenwald. International vergleichende Messung von Beschäftigung und Arbeitslosigkeit Bestrebungen zur Harmonisierung in der Europäischen Union. In Stat. B. A., Hrsg., Statistische Informationen zum Arbeitsmarkt - Konzept und Kritik, Anwendung und Auslegung, Schriftenreihe Forum der Bundesstatistik, Band 30. Metzler-Poeschel Verlag, Stuttgart, 1997. 
Sachverständigenrat zur Begutachtung der gesamtwirtschaftlichen Entwicklung. Vor weitreichenden Entscheidungen, Jahresgutachten 1998/99. Metzler-Poeschel Verlag, Stuttgart, 1998.

R. Schettkat. Jobwunder Niederlande. MitAB, 4, S. 807-810, 1997.

G. Schmid. Arbeitslosigkeit und Beschäftigung in Europa: Ansätze zu einer Theorie des Beschäftigungsregimes. In: Stat. B. A., Hrsg., Statistische Informationen zum Arbeitsmarkt - Konzept und Kritik, Anwendung und Auslegung, Schriftenreihe Forum der Bundesstatistik, Band 30. Metzler-Poeschel Verlag, Stuttgart, 1997.

C. Sorrentino. International comparisons of unemployment indicators. Monthly Labour Review, March, S. 3-24, 1993.

C. Sorrentino. International unemployment indicators, 1983-93. Monthly Labour Review, August, S. 31-50, 1995.

Statistisches Bundesamt. Methoden, Verfahren, Entwicklungen, Ausgabe 1, 1997.

Statistisches Bundesamt. Stand und Entwicklung der Erwerbstätigkeit 1997, Ergebnisse des Mikrozensus. Fachserie, 1, Reihe 4.1.1. Stuttgart, 1998.

D. Steel. Producing Monthly Estimates of Unemployment and Employment According to the International Labour Office Definition (with discussion). J.Roy.Statist.Soc., Ser. A, S. 5-46, 1997.

L. Tronti (Ed.). Benchmarking Employment Performance and Labour Market Policies, Final Report 1997. I.A.S., Institute for Applied Socio-Economics, Berlin, 1998.

J. Velling. Wie hoch ist die Arbeitslosigkeit in Deutschland wirklich. ZEW-Newsletter, Nr. 2, Dezember, S. 10-14, 1994.

H. Werner. Unterschiede in der Erfassung der Arbeitslosigkeit. MittAB, 1982.

A. White and J. Leyland. How unemployment is measured in different countries. Employment Gazette, September, S. 421-431, 1992.

Working Party on the Measurement of Unemployment in the UK. The measurement of unemployment in the UK (with discussion). J.Roy.Statist.Soc., Ser. A., S. 363-417, 1995. 
Adresse des Autors:

apl.Prof. Dr. Hans Peter Litz

Institut für Volkswirtschaftslehre II und Statistik

Carl von Ossietzky Universität Oldenburg

Ammerländer Heerstraße 114-118

D-26129 Oldenburg, Deutschland

Tel.: +49 441 / 798-8303(8227)

Fax: $+49441 / 798-8228$

Email: hans.p.litz@uni-oldenburg.de 